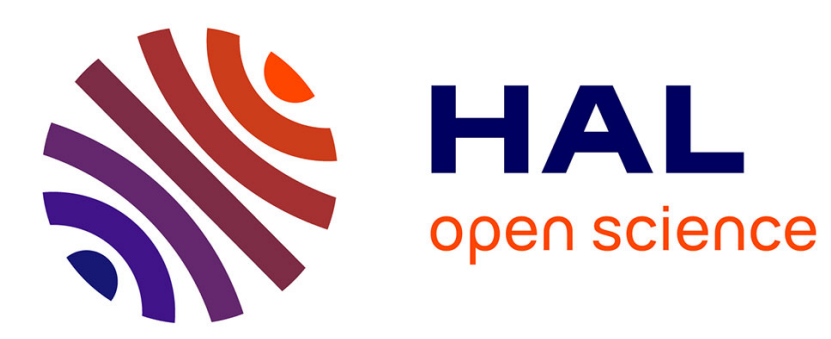

\title{
La relation Homme-Animal. Conséquences et possibilités d'amélioration
}

\author{
Marie-France Bouissou
}

\section{To cite this version:}

Marie-France Bouissou. La relation Homme-Animal. Conséquences et possibilités d'amélioration. Productions Animales, 1992, 5 (5), pp.303-318. hal-00895986

\section{HAL Id: hal-00895986 https://hal.science/hal-00895986}

Submitted on 1 Jan 1992

HAL is a multi-disciplinary open access archive for the deposit and dissemination of scientific research documents, whether they are published or not. The documents may come from teaching and research institutions in France or abroad, or from public or private research centers.
L'archive ouverte pluridisciplinaire HAL, est destinée au dépôt et à la diffusion de documents scientifiques de niveau recherche, publiés ou non, émanant des établissements d'enseignement et de recherche français ou étrangers, des laboratoires publics ou privés. 
INRA Prod. Anim., 1992,5 (5), 303-318.
Marie-France BOUISSOU

INRA Station de Physiologie de la Reproduction 37380 Nouzilly
La relation Homme-Animal. Conséquences et possibilités d'amélioration
L'animal est en interaction continue avec son environnement et toute perception d'un changement entraîne chez lui une réaction physiologique ou comportementale. Le milieu dans lequel sont entretenus nos animaux domestiques est très particulier (alimentation rationnée et localisée, espace limité, environnement social modifié, etc...) de plus l'Homme l'influence de manière importante. Tout au long de sa vie, l'animal domestique a plus ou moins de contact avec l'Homme selon son espèce et son mode d'élevage (intensif ou extensif). Parmi ces contacts, certains sont de nature positive, en particulier l'apport d'aliment, mais aussi la traite et certains soins corporels (par exemple l' entretien du pelage), mais dans leur grande majorité, les contacts sont de nature aversive : castration, transports, traitements sanitaires, tonte, écornage, etc... La simple présence d'un humain peut même être source de peur pour un animal non familiarisé. Le type de réaction induit par l'Homme dépend de la nature du contact mais aussi de la qualité de la relation existant

\section{Résumé}

La qualité de la relation Homme-Animal et le comportement de l'éleveur sont des facteurs importants à prendre en compte dans les différents systèmes de production, car ils ont un impact sur la productivité, la santé et le bien-être des animaux.

Une mauvaise qualité de la relation Homme-Animal se traduit en particulier par des réactions de peur des animaux. Différentes méthodes ont été proposées pour évaluer ces réactions en présence d'un humain ; elles reposent sur des critères comportementaux (distance de fuite, latence d'approche, temps passé à proximité, réaction à la contention, etc...) et physiologiques (fréquence cardiaque, cortisolémie). La qualité de la relation Homme-Animal est influencée par les conditions d'élevage, d'entretien, la personnalité et l'attitude de l'éleveur, l'expérience de l'animal, la nature des contacts antérieurs et enfin par le patrimoine génétique. Elle a pour conséquence des réactions de stress qui, à leur tour, entraînent des baisses de performances dans des domaines divers (production, reproduction).

Il est possible d'améliorer la relation Homme-Animal en modifiant l'attitude de l'éleveur, par des techniques d'habituation, d'apprentissage, par l'établissement de liens privilégiés grâce à des manipulations neutres ou plaisantes à des périodes particulières de la vie de l'animal.ur l'avoine et le sorgho. entre l'Homme et l'animal : peur ou au contraire relation de confiance, lien social.

Enfin, les techniques modernes d'élevage se caractérisent souvent par la constitution de groupes d'animaux de taille importante et par la mécanisation et la simplification des différents traitements (alimentation, traite, nettoyage des installations...) Il en résulte une réduction extrême des contacts de l'éleveur avec le bétail. C'est ainsi, par exemple, qu'un porc de $100 \mathrm{~kg}$ ne nécessite, pour son entretien et celui de sa mère pendant la gestation, qu'une heure de main-d'œuvre.

Il résulte de tout ceci une absence de familiarisation entre l'Homme et l'animal voire même, le plus souvent, des réactions de peur intenses de ce dernier (cf. revue de Hemsworth et Barnett 1987). En conséquence, les manipulations nécessaires peuvent occasionner divers problèmes qui vont de la simple perte de temps aux accidents corporels parfois graves tant pour l'animal que pour l'éleveur.

$\mathrm{Au}$ cours de ces dernières années, l'intérêt pour l'étude de la relation Homme-Animal chez les espèces domestiques s'est accru, en particulier pour des raisons économiques. En effet, une mauvaise qualité de cette relation a des conséquences néfastes sur la productivité : une diminution de la production de lait chez les bovins (Seabrook 1972) et les caprins (Lyons 1989), une baisse de la capacité de reproduction chez les porcins (Hemsworth et al 1981, 1989) ainsi que des réactions de stress qui se traduisent par une diminution de la vitesse de croissance chez le jeune porc (Hemsworth et al 1981), une diminution de la fertilité chez la truie, et une réduction de la taille des testicules chez le verrat (Hemsworth et al 1986).

Le traitement subi par les animaux de boucherie avant l'abattage constitue l'une des expériences les plus stressantes qui soient. De nombreux facteurs interviennent tels que le mélange d'animaux étrangers, le transport, le jeûne, des manipulations brutales (en particulier l'usage de l'aiguillon électrique), des 
installations inadaptées, le temps passé dans les différentes parties de la chaîne d'abattage (Cockram et Corley 1991, Warris 1990). L'ensemble des agents stressants entraîne des réactions comportementales et physiologiques qui affectent la qualité de la viande (cf. revue de Warris 1990). Grandin rapportait en 1980 qu'aux U.S.A les pertes économiques dues aux blessures et meurtrissures consécutives à des manipulations brutales au cours des transports et dans les abattoirs, sont de l'ordre de 46 millions de dollars par an en ce qui concerne les bovins, ovins et porcins. Des manipulations effectuées correctement peuvent permettre de réduire ces pertes de manière substantielle.

Enfin, l'opinion publique se préoccupe de plus en plus du bien-être des animaux d'élevage. Une partie de ce bien-être passe par une bonne relation avec l'éleveur.

Il est donc essentiel, non seulement de prendre en compte la qualité de la relation Homme-Animal dans les techniques d'élevage, mais également de développer des méthodes susceptibles de l'améliorer.

\section{1 / Evaluation de la relation Homme-Animal}

Pour Metz (1987), de nombreux effets négatifs de la relation Homme-Animal sont liés au fait que l'éleveur induit chez ce dernier des réactions de peur par le simple fait de sa présence. C'est donc surtout l'évaluation de la peur de l'Homme qui a été tentée. Ceci peut présenter un double intérêt : au plan de l'étude du phénomène lui-même et de ses composantes, mais aussi comme mesure "clinique" permettant par exemple d'évaluer, sur le terrain, l'efficacité d'un système d'élevage.

Bien que les phénomènes de peur aient fait l'objet de nombreux travaux, ils n'en restent pas moins difficiles à reconnaître et à mesurer voire même à définir (cf revues de Archer 1979, Jones 1987b).

Il est généralement admis que la fonction des réponses de peur est de protéger l'individu d'un danger potentiel. Partant de l'hypothèse que la crainte d'un stimulus effrayant s'exprime par une réticence à s'en approcher ou une réaction d'évitement, le taux d'évitement ou au contraire d'approche d'un être humain sont des éléments utiles pour évaluer la peur induite par l'Homme. La plupart des méthodes de mesure sont effectivement basées sur la distance de fuite vis-à-vis d'un homme en mouvement, la réaction à des tentatives d'attouchement, le temps et la latence d'alimentation en présence d'un humain. De leur côté, Hemsworth et al (1981a) ont mis au point, pour les porcs, une épreuve standardisée dans laquelle ils mesurent la latence d'approche, le temps passé à moins de $50 \mathrm{~cm}$ d'un humain immobile, ainsi que les éventuels contacts effectués par l'animal avec l'expérimentateur. Les réactions comportementales peuvent être également évaluées lors de la capture et de la contention ou des tentatives d'isolement des animaux (tableau 1).

Outre ces indices comportementaux, la mesure de l'élévation du taux plasmatique de corticostéroïdes en présence de l'Homme ou après diverses manipulations a été largement utilisée dans l'appréciation de la peur et des réponses de stress. Chez le Porc, il y a une corrélation positive entre le temps mis par un animal pour s'approcher à moins de $50 \mathrm{~cm}$ d'un humain et pour entrer en contact avec lui et la réponse corticosurrénalienne (Hemsworth et Barnett 1988). La corrélation entre ces deux types de mesures permet de les considérer comme de bons indices du niveau de peur visà-vis de l'Homme.

L'élévation de la fréquence cardiaque est également un paramètre qui a été fréquemment utilisé chez les bovins, les ovins, les chevaux, les porcs, les chèvres, les poules. Cependant, la corrélation entre les réponses comportementales et physiologiques (en particulier la fréquence cardiaque) ne sont pas toujours en accord (Jones et al 1981, Lyons et Price, 1987, Baldock et Sibly 1990 ; tableau 1).

\section{2 / Facteurs influençant la relation Homme-Animal}

\section{1 / Les conditions d'élevage au cours du jeune âge}

Il est bien connu qu'une des meilleures façons de familiariser un jeune animal est de le séparer de sa mère, voire de tout congénère, et de l'élever artificiellement. Parfois, comme c'est le cas chez la Chèvre, outre la réduction de peur vis-à-vis des humains, on obtient aussi une moins grande réactivité vis-à-vis de situations nouvelles (Lyons 1987 ; Lyons et al 1988, Lyons 1989).

Des cas extrêmes de formation de liens interspécifiques, qui peuvent s'apparenter à "l'empreinte", ont été décrits chez des ongulés domestiques (Sambraus 1975). Ce type de relation, conduisant à une trop grande familiarité des animaux, n'est cependant pas souhaitable à cause des perturbations comportementales qui peuvent en découler, et des risques d'accidents en ce qui concerne les espèces de grande taille. Price et Wallach (1990) ont montré que les taureaux élevés en isolement sont plus agressifs vis-à-vis des humains que ceux élevés en groupe. Cette agressivité accrue serait due à la fois à une réduction de la peur de l'Homme et au fait que les animaux n'ont pu apprendre l'expression normale des comportements de soumission. La réputation de plus grande agressivité des taureaux de race laitière pourrait s'expliquer par le fait qu'ils sont généralement élevés artificiellement en case individuelle. 
Tableau 1. Évaluation de la peur vis-à-vis de l'Homme.

\begin{tabular}{|c|c|c|}
\hline Méthodes & Espèces & Références \\
\hline \multicolumn{3}{|l|}{ Critères comportementaux } \\
\hline \multirow{2}{*}{ Distance de fuite } & Chevaux & Mc Cann et al 1988 \\
\hline & $\begin{array}{l}\text { Bovins } \\
\text { Ovins }\end{array}$ & $\begin{array}{l}\text { Murphey et al } 1980 \\
\text { Boissy et Bouissou } 1988 \\
\text { Hutson } 1982\end{array}$ \\
\hline Latence d'approche & Porcins & $\begin{array}{l}\text { Hemsworth et Barnett } 1987 \\
\text { Hemsworth et al } 1991\end{array}$ \\
\hline $\begin{array}{l}\text { Réactions aux tentatives } \\
\text { d'attouchement }\end{array}$ & Bovins & Kerr et WoodGush 1987 \\
\hline $\begin{array}{l}\text { Latence et temps d'alimentation } \\
\text { en présence d'un humain }\end{array}$ & Bovins & $\begin{array}{l}\text { Sato et al } 1984 \\
\text { Boissy et Bouissou } 1988\end{array}$ \\
\hline \multirow[t]{3}{*}{$\begin{array}{l}\text { Réaction à la capture, } \\
\text { contention, tentative } \\
\text { d'isolement }\end{array}$} & Bovins & $\begin{array}{l}\text { Hearshaw et al } 1979,1984 \\
\text { Fordyce et al } 1985 \\
\text { Goddard et al } 1985 \\
\text { Veissier } 1987 \\
\text { Boissy et Bouissou } 1988 \\
\text { Boivin et al } 1992\end{array}$ \\
\hline & Ovins & Mateo et al 1991 \\
\hline & Poulets & $\begin{array}{l}\text { Murphy et Duncan } 1977 \\
\text { Jones et Faure } 1981 \\
\text { Jones 1987b }\end{array}$ \\
\hline \multicolumn{3}{|l|}{ Critères physiologiques } \\
\hline \multirow{3}{*}{$\begin{array}{l}\text { Élévation de la cortisolémie } \\
\text { en présence d'un humain } \\
\text { ou après manipulations }\end{array}$} & Porcins & Hemsworth et al 1981, 1986, 1987 \\
\hline & $\begin{array}{l}\text { Bovins } \\
\text { Ovins }\end{array}$ & $\begin{array}{l}\text { Hemsworth et al } 1987,1988 \\
\text { Boissy et Bouissou } 1988 \\
\text { Fell et Shutt } 1989 \\
\text { Hargreaves et Hutson } 1990\end{array}$ \\
\hline & Caprins & Lyons et al 1988 \\
\hline \multirow{6}{*}{$\begin{array}{l}\text { Élévation de } \\
\text { la fréquence cardiaque }\end{array}$} & Bovins & Boissy et Bouissou 1988 \\
\hline & Ovins & $\begin{array}{l}\text { Hargreaves et Hutson } 1990 \\
\text { Baldock et Sibly } 1990\end{array}$ \\
\hline & Chevaux & Mc Cann et al 1988 \\
\hline & Porcins & Seabrook et Bartle 1991 \\
\hline & Caprins & Lyons et Price 1987 \\
\hline & Poulets & Jones et al 1984a \\
\hline
\end{tabular}

\section{2 / Les conditions d'entretien}

Les conditions d'entretien de l'animal affectent sa réaction aux manipulations. Ainsi, des ovins élevés en bergerie et qui côtoient fréquemment des humains, présentent des réactions physiologiques moindres lors de manipulations que des animaux élevés au pâturage (Reid et Mills 1962).

Des porcs élevés dans un environnement enrichi (présence d'objets à manipuler) et en contact régulier avec des humains sont plus faciles à manipuler, à conduire, et à faire entrer dans un appareil de contention que des animaux élevés sans stimulation particulière
(Grandin et al 1984, Grandin et al 1987). De plus, la fourniture de tels objets réduit la peur des humains quel que soit le type de manipulations (plaisantes ou aversives) qu'ils sont amenés à faire subir aux animaux (Pearce et al 1989). Chez la poule pondeuse, les animaux occupant la rangée du haut dans des batteries superposées, sont plus craintifs vis-à-vis de l'Homme et dans d'autres situations effrayantes, et ont une durée d'immobilité tonique (c'est-à-dire une inhibition motrice temporaire induite par la contention) supérieure à celle d'animaux logés dans la rangée inférieure. Ces différences sont attribuées à un contact réduit avec les humains (Jones 1985, Hemsworth et Barnett 1987). 


\section{3 / La personnalité et l'attitude de l'éleveur}

Il y a deux aspects dans les aptitudes d'une personne à s'occuper d'animaux : un aspect professionnel, pour lequel les connaissances et l'expérience sont importantes, et un aspect que l'on peut qualifier de personnel qui fait que, à qualification technique égale, certains individus ont plus de "talent" pour tel ou tel aspect de l'élevage. Les capacités de juger, anticiper, entendre, sentir au sens propre et figuré interviennent et sont influencées par le psychisme et les intérêts des sujets. Certaines personnes sont, de par leurs caractéristiques psychologiques, incapables d'exploiter le potentiel génétique des animaux dont ils s'occupent ; la réduction de production laitière peut, dans certains cas, atteindre $1500 \mathrm{~kg} / \mathrm{vache} / \mathrm{an}$ par rapport à des animaux entretenus par de "bons" éleveurs (figure 1, Seabrook 1984). Selon Schlichting et Smidt (1987), le trayeur influence à la fois la production laitière et la teneur du lait en matières grasses. Les personnes introverties et sûres d'elles sont celles qui obtiennent les meilleurs résultats (Seabrook 1984). Celles qui sont sûres d'elles mêmes, calmes et bien équilibrées ont également moins de problèmes avec les taureaux d'insémination (Sambraus et Unshelm 1983). Hemsworth et al (1989) ont montré que certaines caractéristiques de la personnalité et du comportement de l'éleveur étaient de bons prédicteurs du niveau de peur des truies. Le niveau de peur des humains est par ailleurs fortement corrélé aux performances reproductrices (taux de gestation, taille de portée, etc...).

La connaissance précise des réactions individuelles des animaux et les capacités d'observation de l'éleveur rendent possible la détection de changements très discrets. Ainsi, il existe des différences dans l'aptitude à détecter l'oestrus. Ceci peut conduire à des échecs de la reproduction et donc à des pertes économiques (Jensen 1986).

\section{4 / L'expérience passée et la nature des contacts}

Le niveau de peur est grandement influencé par l'expérience de l'animal et la nature des contacts qu'il a eu précédemment avec l'Homme. Dans le cas de contacts aversifs (par exemple un choc électrique à chaque tentative d'approche), l'animal apprend rapidement à éviter ultérieurement les humains ; inversement, si le contact est positif (de type caresses) la peur des humains est alors réduite (Hemsworth et al 1981).

Des ovins australiens ayant subi l'opération du "mulesing" qui consiste à enlever la laine et la peau de la région postérieure pour éviter les myiases (développement de larves de Diptères) présentent une forte réaction d'évitement visà-vis de la personne qui les a tenus durant l'opération, et ce pendant plus de 5 semaines (figure 2). Cette réaction est très spécifique car les animaux opérés ne montrent pas de crainte vis-à-vis d'autres humains (en particulier celui
Figure 1 Influence du vacher sur la production laitière (Seabrook 1984).
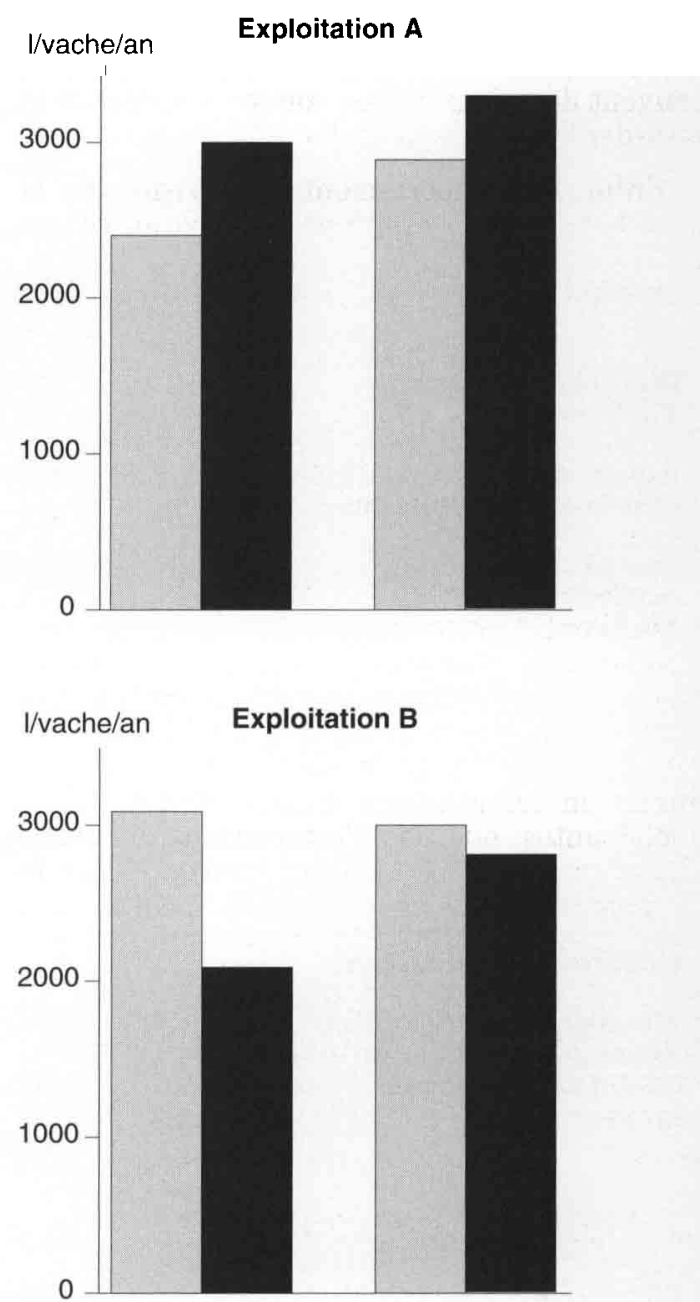

Moyenne des 2 ans avant le changement de vacher

Moyenne des 2 ans suivant le changement

chargé de les soigner), ni d'un environnement nouveau en l'absence d'humain (Fell et Shutt 1989).

Une expérience négative peut avoir une influence à long terme et rendre plus difficile la manipulation des animaux : des moutons ayant été maintenus dans un appareil de contention qui les renverse sur le dos sont, un an après, plus difficiles à faire passer d'un enclos à un autre (Hutson 1985).

Mêmes certains types de contacts ou attitudes humaines inoffensives, que l'on pourrait a priori penser sans conséquences négatives, peuvent influencer de manière nette la réaction vis-à-vis de l'Homme ; par exemple, un homme debout, portant des gants, tentant de s'approcher ou d'entrer en contact physique, est perçu par les porcs comme plus menaçant qu'un humain accroupi, mains nues et immobile. De manière plus surprenante, l'exposition des animaux à ces types de comportements réduit le taux de croissance de jeunes porcs 
dans les mêmes proportions qu'une manipulation réellement aversive comme un choc électrique (Hemsworth et al 1986 ; Gonyou et al 1986). De nombreux signaux émis par l'Homme, volontairement ou inconsciemment peuvent donc être perçus comme menaçants et retarder l'établissement d'une bonne relation.

Enfin, un comportement imprévisible de la part de l'éleveur, qui comporte ne serait-ce que $20 \%$ de manipulations aversives et $80 \%$ de manipulations positives, accroît fortement la peur des humains chez le Porc, et provoque de plus un stress chronique et une réduction de la croissance des animaux. De fréquentes manipulations plaisantes ne masquent donc pas les effets négatifs de manipulations aversives même peu fréquentes (Hemsworth et al 1987b).

Cependant, Dryden et Seabrook (1986) ont montré que chez la truie, des interactions avec l'Homme, qu'elles soient plaisantes ou déplaisantes, entraînent chez leurs porcelets des taux de croissance supérieurs à ceux des témoins. Les auteurs concluent qu'il vaut mieux un certain taux d'interactions, même déplaisantes, que pas d'interactions du tout ; elles diminueraient l'ennui provoqué par lé manque de stimulations dans les cages de mise bas, réduisant ainsi le stress subi par les truies, ce qui conduirait à une production laitière supérieure. Par ailleurs, Hargreaves et Hutson $(1990 d)$ ont remarqué que la répétition d'un traitement aversif (tonte simulée) avait néanmoins un effet d'apprivoisement chez les ovins.

\section{5 / Influences génétiques}

L'adaptation des animaux aux divers types de production impliquant des degrés divers d'intervention humaine dépend vraisemblablement en partie de leur réactivité vis-à-vis de l'Homme. Cette réactivité peut dépendre de facteurs génétiques : on sait, en effet, que la souche ou la race influencent le "tempérament", la réactivité et la facilité d'approche par l'Homme chez le Poulet (Murphy et Duncan 1978), les bovins (Hearnshaw et al 1979, 1984, Adeyemo et Heath 1982, Murphey et al 1980, 1981), les ovins (Grandin 1987, Romeyer et Bouissou 1992), et les porcins (Mormède et al 1984). Ainsi, par exemple, les bovins de races laitières sont plus faciles à approcher et à manipuler que ceux des races à viande et ce, quel que soit leur mode d'élevage (Murphey et al 1980). On peut penser qu'une sélection consciente ou non sur la facilité de manipulation a eu lieu en même temps que celle sur l'aptitude laitière.

Lyons et al (1988a) ont séparé à la naissance des couples de chevrettes jumelles ; les unes ont été laissées avec la mère, les autres ont été élevées artificiellement par l'Homme. A l'âge de sept mois, tous les animaux ont été soumis à différentes épreuves en présence d'un homme. Hormis le fait que les animaux manipulés présentent des réactions de peur envers l'homme plus faibles que les témoins, les auteurs ont constaté que le classement d'un
Figure 2 Réaction des ovins, ayant subi ou non l'opération de "mulesing", à la présence d'un humain (Fell et Shutt 1989).

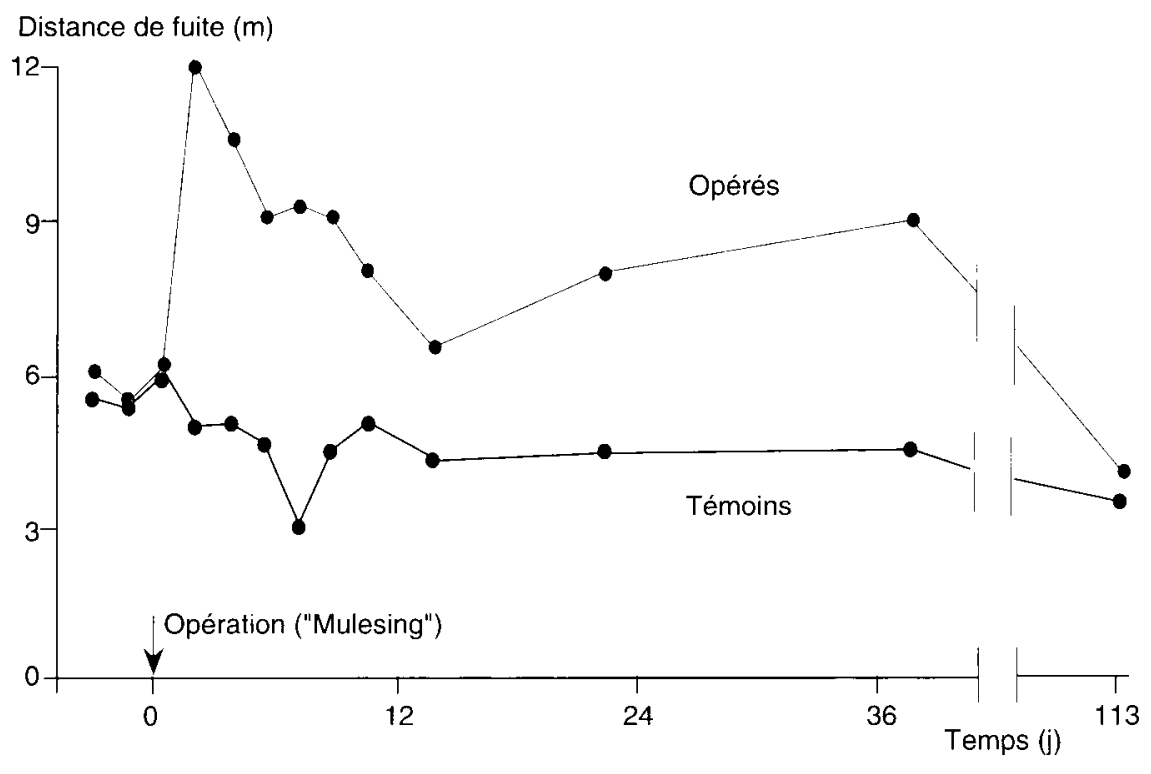

individu dans le groupe élevé par la mère permet de prédire celui de sa soeur jumelle dans le groupe élevé artificiellement.

Les réactions des renardeaux vis-à-vis de l'Homme sont positivement corrélées à celles de leurs mères (Braastad et al 1989). Dans cette espèce Belyaev et al $(1984,1985)$ ont d'ailleurs pu effectuer une sélection sur l'attitude de renards argentés vis-à-vis de l'Homme et obtenir deux populations totalement différentes, l'une docile, l'autre agressive (non sélectionnée).

Récemment, Hemsworth et al (1990) ont montré chez le Porc, que certaines composantes des réactions de peur vis-à-vis de l'Homme (par exemple la latence de la première interaction au cours d'épreuves standard) sont modérément héritables et pourraient donc donner prise à la sélection.

Le facteur génétique joue donc un rôle important dans la relation Homme-Animal.

\section{3 / Conséquences économiques d'une mauvaise relation Homme-Animal}

Outre les réactions d'évitement marquées vis-à-vis de l'Homme qui rendent les manipulations difficiles et entraînent des risques d'accidents tant pour l'animal que pour l'éleveur, une mauvaise relation entraîne des conséquences défavorables dans divers domaines.

Chez le Porc, de nombreux travaux ont montré que le niveau de peur des animaux vis-à-vis des humains est corrélé négativement avec les performances de reproduction (tableau 2).

De plus, les animaux qui subissent des manipulations aversives, par exemple un choc 
électrique à chaque tentative d'approche, présentent un état de stress chronique indiqué par une élévation du taux de corticoïdes (Hemsworth et al 1987), qui se répercute au niveau de la production : la vitesse de croissance chez le jeune est réduite, l'âge au premier oestrus est inférieur car les femelles sont plus sensibles à un stress aigu et donc plus réactives à la stimulation par le verrat, le taux de gestation chez la femelle adulte est inférieur, le nombre de porcelets nés vivants est inférieur et la mortalité à 3 semaines est supérieure, les mâles présentent un comportement copulatoire adapté plus tardivement, et leur taille testiculaire à 23 semaines est inférieure (figure 3) (Hemsworth et al 1981, 1986a, b, Gonyou et al 1986, Barnett et al 1982/1983, Dryden et Seabrook 1986, Paterson et Pearce 1989, Pearce et al 1989, Hemsworth et al 1981). L'ampleur de ces baisses de performances est parfois surprenante et peut aller jusqu'à $11,3 \%$ de réduction en ce qui concerne la vitesse de croissance, et $62 \%$ en ce qui concerne le taux de gestation (Hemsworth et Barnett 1987).

Chez le Poulet, des manipulations très aversives, comme des prélèvements sanguins par ponction intracardiaque entraînent une diminution du poids corporel à 8 semaines (Buckland et al 1974).

Chez les vaches, le changement de trayeur ou le fait qu'il soit perturbé par des bruits ou des insomnies peut entraîner une baisse de la production laitière (figure 4 )(Schlichting et Smidt 1987, Seabrook 1987).

La comparaison de troupeaux laitiers à forte ou au contraire à faible production laitière montre que dans ces derniers la relation Homme-Animal est moins bonne : les animaux entrent moins facilement en salle de traite et y défequent plus, ont une distance de fuite supérieure, approchent moins facilement volontairement un humain, le trayeur leur parle moins et les touche moins (figure 5) (Seabrook 1984).

Nous avons déjà mentionné les pertes importantes qui résultent des meurtrissures et

Tableau 2. Conséquences du type de contact avec l'homme chez le porc.

\begin{tabular}{|c|c|c|}
\hline Contact & Effets sur les animaux & Références \\
\hline Plaisant & $\begin{array}{l}\text { - plus calmes } \\
\text { - faciles à approcher } \\
\text { - plus grand nombre de } \\
\text { porcelets } \\
\text { - vitesse de croissance } \\
\text { des jeunes supérieure }\end{array}$ & $\begin{array}{l}\text { Hemsworth et al } 1981 \\
\text { Dryden et Seabrook } 1986 \\
\text { Hemsworth et al } 1981 \\
\text { Dryden et Seabrook } 1986\end{array}$ \\
\hline Déplaisant & $\begin{array}{l}\text { - plus agités } \\
\text { - moins faciles à approcher } \\
\text { - fertilité réduite } \\
\text { - cortisolémie élevée }\end{array}$ & $\begin{array}{l}\text { Hemsworth et al } 1981 \\
\text { Dryden et Seabrook } 1986 \\
\text { Hemsworth et al } 1981\end{array}$ \\
\hline
\end{tabular}

Figure 3. Effet du type de manipulations chez le porc (Hemsworth et al 1986, Hemsworth et Barnett 1987).
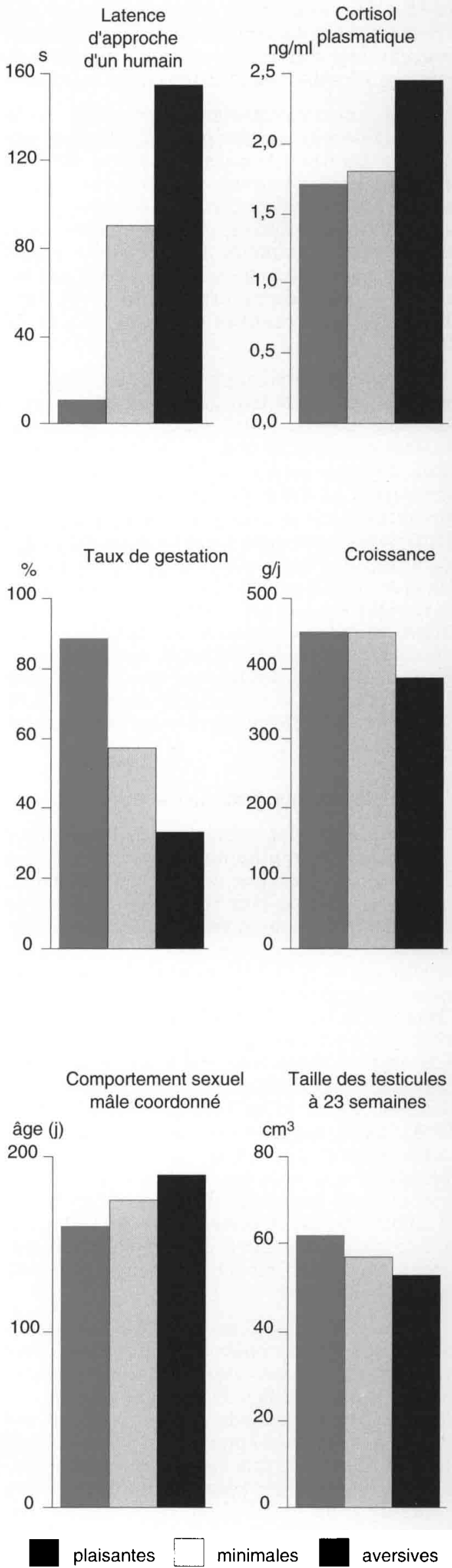
Figure 4. Effet du comportement du vacher sur la production laitière du troupeau (Seabrook 1987). $\mathrm{I} / \mathrm{j}$

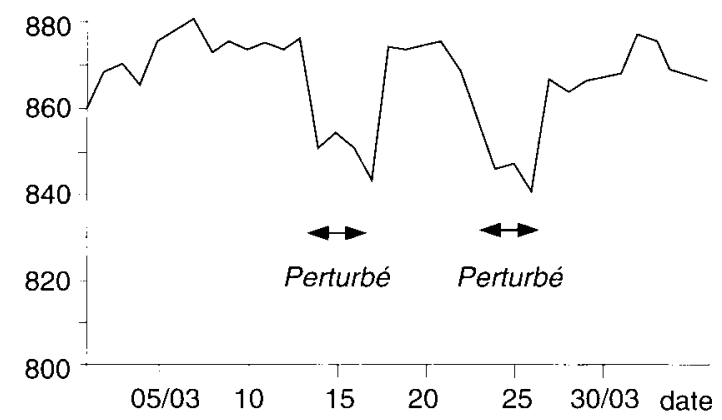

blessures subies par les bovins lors des transports et des manipulations précédant l'abattage. Des changements physiologiques liés au stress entraînent également des modifications de la qualité de la viande. Pour une bonne part, ces problèmes sont dûs à des manipulations brutales et à une méconnaissance du comportement et des capacités sensorielles des animaux. Grandin (1984/1985) a proposé des dispositifs qui prennent en compte ces facteurs et facilitent la manipulation des animaux, en particulier dans les abattoirs, permettant ainsi une réduction du stress subi, et par là-même des pertes économiques

\section{4 / Possibilités d'améliorer la relation Homme-Animal}

Outre la maîtrise des différents facteurs évoqués précédemment et qui influencent la qualité de la relation Homme-Animal, de nombreux auteurs ont tenté de développer des techniques pour améliorer cette relation, et en particulier de réduire la peur des animaux face aux humains, ou de faciliter leur manipulation par divers moyens.

\section{1 / Habituation}

L'habituation survient à la suite de la répétition d'un stimulus. Elle peut intervenir lors de manipulations lorsque l'animal apprend qu'il y a toujours une possibilité d'échapper (Fox 1984) ; elle dépend de la possibilité de contrôle de l'agent stressant, et de son pouvoir aversif.

En utilisant une analyse en composantes principales, Sato et al (1983) ont cherché à déterminer les facteurs contrôlant la facilité de manipulation des bovins : elle est expliquée par 3 facteurs parmi lesquels, outre le tempérament de l'animal, on trouve l'habituation au type de manipulation et l'habituation aux humains. Ceci est à relier à l'observation de Rubio et al (1988) qui ont montré, chez les bovins, que les taux de cortisol en réponse à des manipulations décroissaient avec la répétition.

La tonte constitue l'une des procédures les plus stressantes pour les moutons. Hargreaves et Hutson (1990a, b, c, e) ont exposé des ovins à une tonte simulée à 4 reprises à 2 semaines d'intervalle. Bien que le niveau maximal de cortisol plasmatique ne soit pas affecté, il retourne plus rapidement à un niveau de base lors de la quatrième séance. De plus, la distance de fuite vis-à-vis d'un homme qui approche est réduite chez les animaux ainsi traités par rapport à des animaux non manipulés. Cependant, si le traitement est très aversif (électro-immobilisation) la répétition n'a pas d'effet sur les taux de cortisol, ni sur celui des B-endorphines (Jephcott et al 1986).

\section{2 / Apprentissage}

De même que l'animal apprend rapidement à associer la présence d'un humain aux traitements aversifs que celui-ci peut être amené à lui infliger, il peut également l'associer à des situations positives telles que l'approvisionnement en nourriture, et les réactions de peur peuvent ainsi être réduites.

\section{Figure 5. Comportement des vaches laitières et de l'éleveur dans des troupeaux à forte ou faible} production (Seabrook 1984).

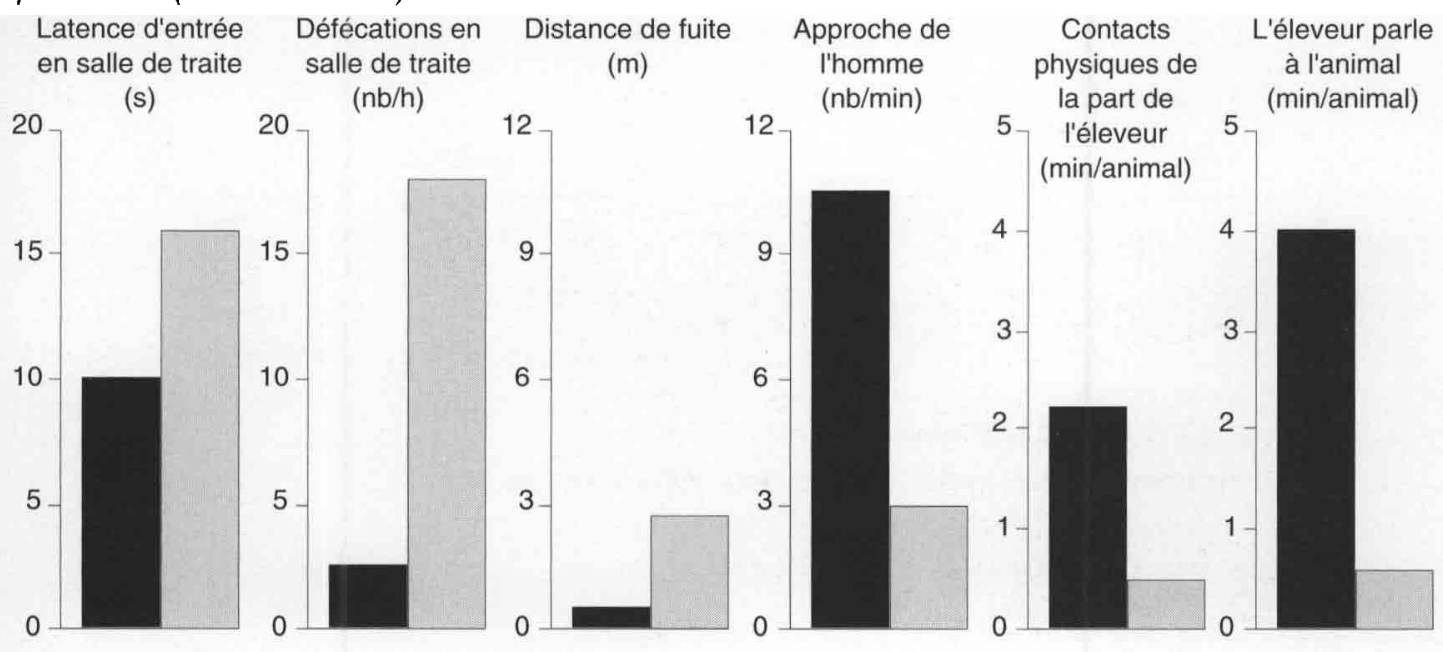

forte production 
Figure 6. Effets d'une récompense alimentaire sur la facilité de manipulation d'ovins soumis à divers types de contention (Hutson 1985).
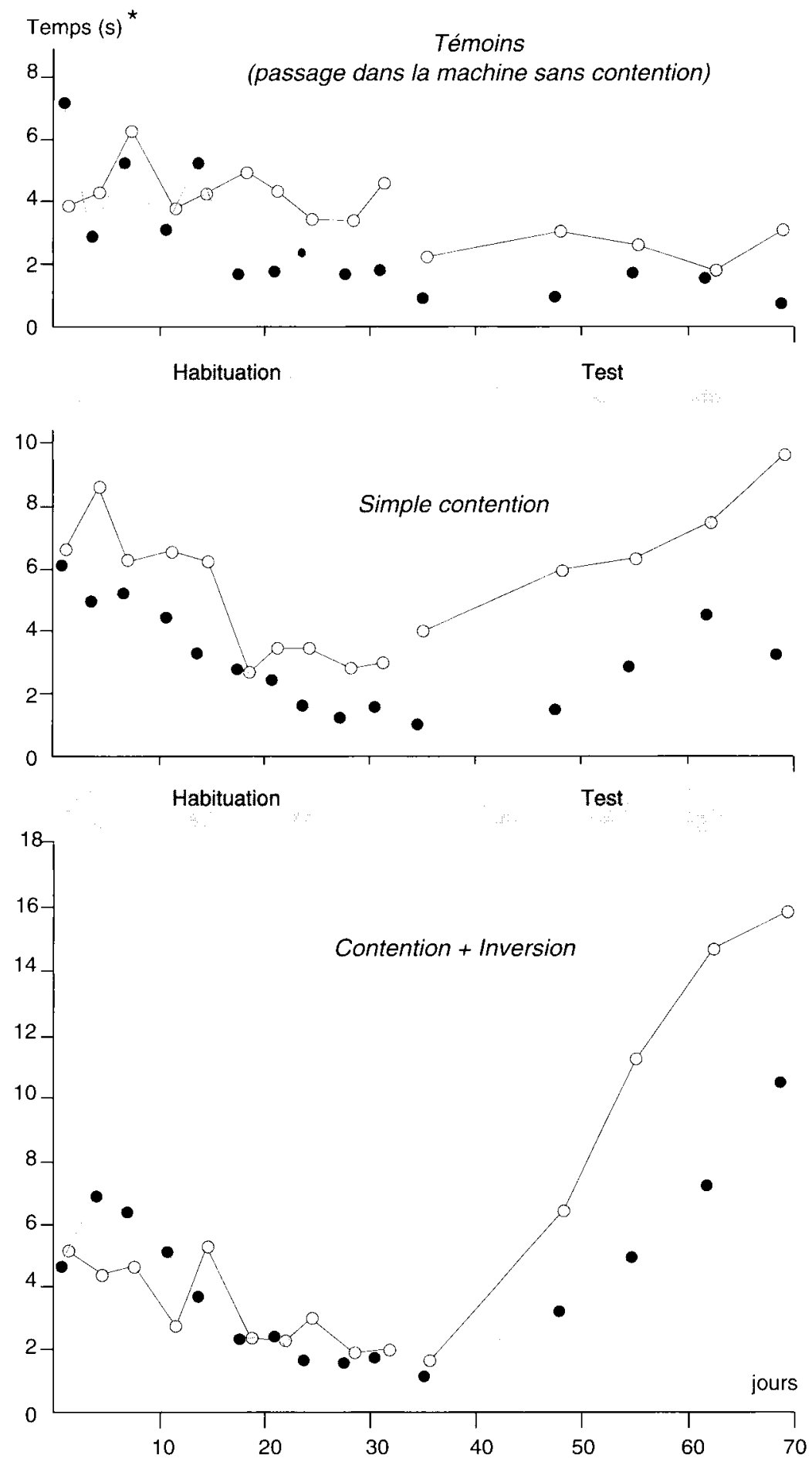

(1 an après)

Habituation
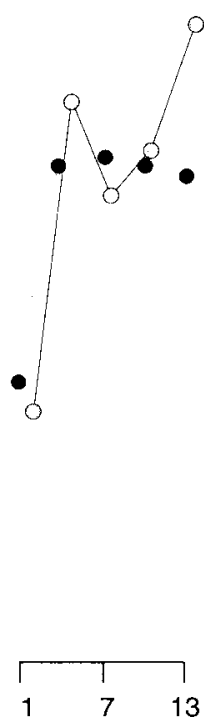

Retest

(1 an après)

- Récompensés

Non récompensés

* Temps nécessaire pour faire entrer l'animal dans un appareil de contention Habituation : 5 secondes dans l'appareil. $(n=10)$

Test : 10 secondes dans l'appareil, avec ou sans contention. $(n=5)$ Retest : test 1 an après. $(n=5)$ 
Une récompense alimentaire associée aux manipulations peut grandement faciliter ces dernières. Le temps nécessaire pour faire entrer des ovins dans un appareil de contention est moindre pour les animaux qui ont été habitués à y recevoir une récompense alimentaire, et ce même un an après (figure 6). Cependant, si le traitement a été très aversif (animaux retournés sur le dos par l'appareil de contention), et bien qu'il y ait une différence lors des premiers tests, elle ne se retrouve plus un an après (Hutson 1985). L'utilisation d'une récompense alimentaire peut même conduire les ovins à entrer spontanément dans un appareil de contention (de type "squeeze-tilt table", Grandin 1989). Il est également bien connu, en pratique, que la distribution de concentré en salle de traite favorise la production ; cet effet ne peut être attribué à un effet nutritionnel car même de très petites quantités sont efficaces. Plus probablement, ce sont les conditions psychologiques positives de l'animal qui en sont responsables.

L'association de la présence humaine avec la nourriture n'a cependant pas d'effet sur des poules adultes d'après Murphy et Duncan (1977) ; elle ne permet d'augmenter le taux d'approche d'un humain que chez des poussins appartenant à une lignée dite "docile".

L'observation d'autres animaux approchant et entrant en contact avec des humains peut réduire la peur. Des chevrettes étudiées dans des tests standardisés vis-à-vis d'un humain passent plus de temps près de l'Homme si elles sont accompagnées d'un adulte familier et surtout de leur propre mère. Les jeunes qui passent moins de temps que la moyenne près de l'Homme sont qualifiés de "timides" : chez ces animaux, les taux de cortisol plasmatique dépendent du comportement de l'adulte avec lequel ils sont testés, et de la nature des liens sociaux avec celui-ci. Si l'adulte (familier ou inconnu) se montre craintif, il y a une élévation du taux de cortisol. Au contraire, des adultes connus ou non, mais non craintifs réduisent la réactivité surrénalienne des jeunes. Seules les propres mères réduisent cette réactivité quel que soit leur comportement. Il n'y a par contre aucune différence en fonction du contexte social dans lequel se déroulent les épreuves chez les chevreaux qualifiés de "non craintifs" (Lyons et al 1988a, b).

L'influence du contexte social sur les réactions de peur se retrouve chez les gallinacés (Jones 1983, 1984a ; Jones et Merry 1988) et les bovins (Boissy et Le Neindre 1990).

\section{3 / Manipulations douces}

Les manipulations sont désignées par les termes anglo-saxons de "handling" ou de "gentling" et ont été utilisées dans les études faites chez les rongeurs depuis une trentaine d'années. Le "handling" consiste simplement à prendre un animal dans son environnement habituel, et à le transporter dans une cage ou boîte voisine pour quelques instants. Dans le cas du "gentling" des éléments supposés plaisants sont fournis tels que caresses, offrande de nourriture, etc... Il faut cependant garder présent à l'esprit que le traitement considéré comme "plaisant" peut en fait être considéré comme indésirable ou déplaisant par l'animal. Effectuées chez de jeunes animaux, généralement avant le sevrage, de telles manipulations entraînent une réduction de la peur de l'Homme et de nombreuses autres conséquences bénéfiques tant sur la résistance à des perturbations émotionnelles que sur les capacités d'apprentissage, la résistance à des agents pathogènes, etc... et en général elles accroissent la capacité des animaux à faire face à des situations stressantes (Denenberg 1963, 1964 ; Daly 1973).

En ce qui concerne les animaux domestiques, la peur de l'Homme peut également être réduite par des manipulations régulières neutres ou supposées plaisantes chez le Poulet, le Porc, les caprins, les chevaux et les bovins (tableau 3 ).

Des manipulations douces (gentling) ont été utilisées chez les ovins comme moyen possible de réduire l'aversion des animaux pour les divers traitements. Effectuées pendant 35 jours sur des animaux adultes elles ont pour effet de réduire la distance de fuite et la réponse cardiaque à l'approche d'un humain. Curieusement, des traitements aversifs comme la tonte simulée ont des effets similaires. Par ailleurs, les manipulations douces ne réduisent pas l'aversion des moutons pour la tonte simulée (Hargreaves et Hutson 1990d).

De plus, les manipulations s'accompagnent d'une augmentation de la vitesse de croissance chez le Poulet et le Porc, d'un accroissement de la résistance aux agents pathogènes chez le Poulet, d'une amélioration des capacités d'accouplement chez le Porc, d'une moindre rétention de lait chez la Chèvre, d'une augmentation des capacités d'apprentissage chez le Cheval et d'une réduction de l'émotivité en général (souvent appréciée par une activité supérieure en environnement nouveau de type open-field, ou l'approche d'un objet nouveau) chez le Porc, le Cheval, le Renard, la Chèvre et le Lapin (tableau 3). Dans cette dernière espèce on remarque également une réduction de l'incidence d'artériosclérose aortique (qui peut aller jusqu'à $60 \%$ ) chez les animaux soumis à un régime riche en cholestérol (Nerem et al 1980).

Dans certains des travaux mentionnés, les manipulations ont été effectuées sur de jeunes animaux. En effet, l'étude de l'ontogenèse des comportements a mis en évidence le rôle fondamental de l'expérience précoce pour moduler le comportement de l'adulte. En ce qui concerne les relations inter-spécifiques, l'expérience précoce permet de familiariser les animaux avec des représentants d'une autre espèce. Par exemple, un rat ne manifeste plus de comportement d'agression vis-à-vis des souris s'il a été élevé en leur présence (Galef 1970). Les brebis expriment généralement de fortes réactions de peur à l'égard de l'espèce canine (Torres-Hernandez et Hohenboken 1979); cependant, si elles ont été élevées en contact avec un chien, non seulement elles ne sont pas 
Tableau 3. Effets des manipulations

\begin{tabular}{|c|c|c|}
\hline Effets & Espèces & Références \\
\hline $\begin{array}{l}\downarrow \text { Peur de l'homme } \\
\text { Facilité de manipulation }\end{array}$ & $\begin{array}{l}\text { toutes } \\
\text { espèces } \\
\text { étudiées }\end{array}$ & $\begin{array}{l}\text { Hughes et Black } 1976 \\
\text { Murphy et Duncan } 1978 \\
\text { Jones et Faure } 1981 \\
\text { Hemsworth et al } 1981,1986 \\
\text { Sato et al } 1984 \\
\text { Fordyce et al } 1985 \\
\text { Heird et al } 1986 \\
\text { Hemsworth et Barnett } 1987 \\
\text { Grandin et al } 1987 \\
\text { Boissy et Bouissou } 1988 \\
\text { Lyons et al } 1988 \\
\text { Mc Cann et al } 1988\end{array}$ \\
\hline$\downarrow$ Émotivé, peur en général & $\begin{array}{l}\text { Bovins } \\
\text { Caprins } \\
\text { Porcins } \\
\text { Chevaux } \\
\text { Poulets } \\
\text { Lapins } \\
\text { Renards }\end{array}$ & $\begin{array}{l}\text { Hemsworth et al } 1986 \\
\text { Tenessen } 1988 \\
\text { Heird et al } 1989 \\
\text { Kersten et al } 1989 \\
\text { Lyons } 1989 \\
\text { Podberseck et al } 1991\end{array}$ \\
\hline Vitesse de croissance & $\begin{array}{l}\text { Poulets } \\
\text { Porcins }\end{array}$ & $\begin{array}{l}\text { Thompson } 1976 \\
\text { Hemsworth et al } 1981 \\
\text { Jones et Hughes } 1981\end{array}$ \\
\hline $\begin{array}{l}1 \text { Résistance aux agents } \\
\text { pathogènes }\end{array}$ & Poulets & Groos et Siegel 1982 \\
\hline $\begin{array}{l}1 \text { Capacité d'accouplement } \\
\lambda \text { Taux de fertilité }\end{array}$ & Porcins & Hemsworth et al 1986 \\
\hline A Capacité d'apprentissage & Chevaux & Heird et al 1986 \\
\hline$\downarrow$ Rétention du lait & Caprins & Lyons et al 1989 \\
\hline
\end{tabular}

effrayées lors de l'exposition à d'autres chiens mais, lorsqu'elles en sont séparées, elles manifestent des réactions aussi intenses que celles provoquées par la séparation d'avec leurs congénères (Cairns et Johnson 1965).

Dans de nombreuses espèces telles que les canidés (chien, renard) ou les ongulés (mouton et chèvre), il existe une période dite de socialisation au cours de laquelle se créent les attachements sociaux intra- et inter-spécifiques (Scott 1978). Chez le Chien, elle se situe entre les âges de trois et douze semaines (Scott 1978) ; si le jeune animal n'a pas de contact avec l'Homme au cours de cette période, il pourra à la rigueur être apprivoisé mais jamais réellement "socialisé". Chez le Renard, la période optimale coïncide avec une phase importante de la maturation visuelle et auditive (Belyaev et al 1984).

Ainsi, la période à laquelle sont effectuées les manipulations des animaux peut être importante.

Chez le Rat, des stimulations tactiles effectuées uniquement pendant la première semaine de vie sont suffisantes pour réduire les comportements émotionnels (Schaefer 1963). Chez la Perdrix, la vue d'un Homme pendant les 48 heures qui suivent l'éclosion est suffisante pour réduire les réactions de peur et accroître les réponses d'approche (Csermely et al 1983). Chez le Lapin, la manipulation limitée aux dix premiers jours de la vie se révèle inefficace, par contre, le même traitement effectué entre le dixième et le vingtième jour se traduit par une diminution des réactions émotives de ces animaux (Metz 1983 ; Kersten et al 1989).

Chez le Chien et le Renard, des manipulations effectuées avant la 8ème semaine conduisent à une réduction de la crainte des humains, un accroissement des conduites exploratoires, et une réduction de la susceptibilité au stress (Fox et Stelzner 1966, Pedersen et Jeppesen 1990).

L'existence, chez les ongulés domestiques, de périodes de sensibilité particulière au cours desquelles la création de liens interspécifiques serait facilitée est d'une importance économique évidente, spécialement si le contact avec les humains peut être réduit à une très courte période tôt après la naissance.

Hemsworth et al (1986) suggèrent effectivement l'existence d'une période sensible au cours des huit premières semaines de vie chez le Porc, mais ceci reste à confirmer. 
Chez les bovins, Boissy et Bouissou (1988) ont étudié l'effet à long terme de manipulations (brossage et conduite au licol) de veaux Frisons élevés artificiellement à différentes périodes de l'ontogenèse. Les animaux étaient manipulés 30 jours à raison de 3 jours/semaine de la naissance à 3 mois ou de 6 à 9 mois, ou également 30 jours mais à raison de 3 jours/mois entre la naissance et l'âge de 9 mois. Des veaux de même âge ne subissant aucune manipulation particulière servaient de témoins. A l'âge de 15 mois, tous les animaux ont été individuellement soumis à une batterie d'épreuves supposées anxiogènes, impliquant ou non la présence d'un homme. Compte-tenu de l'importance connue des périodes néonatales, l'hypothèse était que la manipulation des animaux au cours des trois premiers mois de la vie pourrait être la plus efficace pour améliorer la relation avec l'Homme. En fait, quel que soit leur âge au moment des manipulations, les animaux "manipulés" sont, dans leur ensemble, plus faciles à approcher, à attraper, à conduire au licol, plus calmes à l'attache en présence d'un Homme (figure 7), et s'alimentent plus dans un environnement non familier en présence d'un humain que les animaux non manipulés (témoins). Ils présentent également une moindre élévation de la cortisolémie après les manipulations (figure 8 ). Cependant, les veaux manipulés de la naissance à 3 mois ne diffèrent que rarement des témoins. Par contre, les meilleurs résultats sont obtenus par les animaux manipulés peu fréquemment mais sur une plus longue période (de 0 à 9 mois). De plus, seuls les animaux de ce groupe présentent un rythme cardiaque inférieur après diverses manipulations ou épreuves et moins de réactions de crainte face à une situation effrayante n'impliquant pas l'Homme. Ainsi, il n'apparaît pas de période particulièrement favorable à la création de liens avec l'Homme tôt au cours de l'ontogenèse chez les bovins, ou tout au moins la familiarisation obtenue doit être entretenue régulièrement. L'absence d'effet à long terme de manipulations précoces est également rapportée dans cette espèce par Sato et al (1984).

Figure 8 Elévation de la cortisolémie chez des veaux manipulés à diverses périodes de leur développement. (Boissy et Bouissou, 1988).

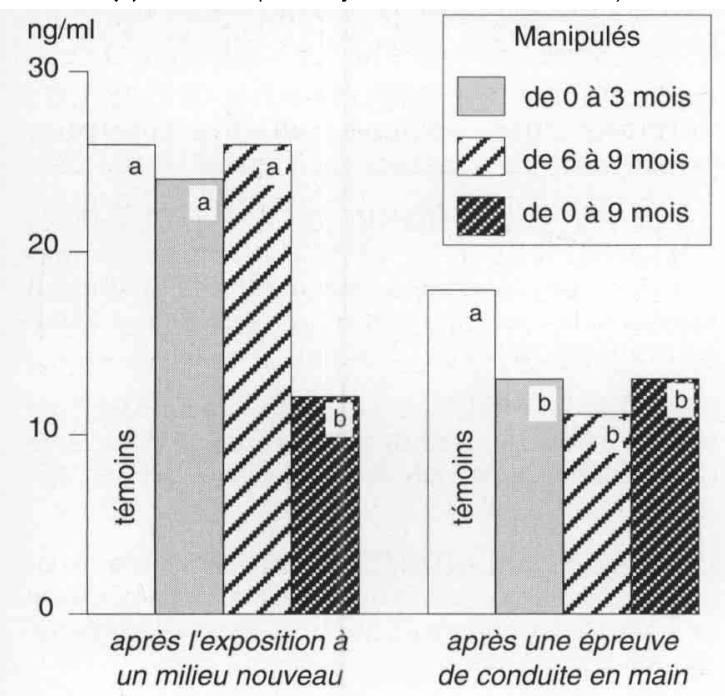

après l'exposition à de conduite en main
Temps nécessaire à la capture des animaux

Comportement à l'attache à proximité d'un humain

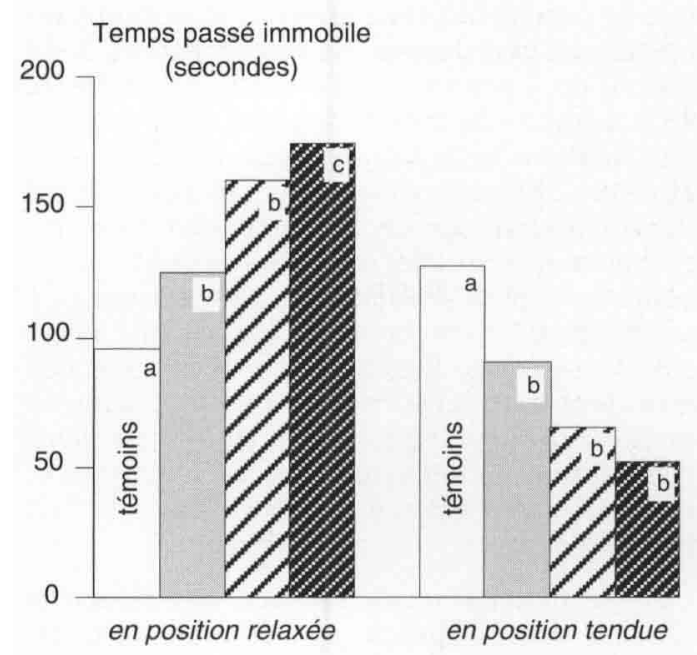

Réactions lors de la conduite au licol
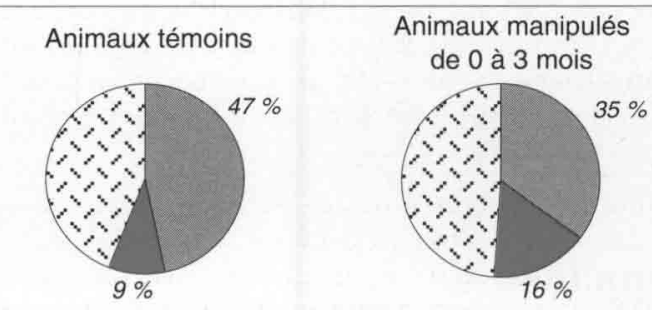

Animaux manipulés de 6 à 9 mois

Animaux manipulés de 0 à 9 mois
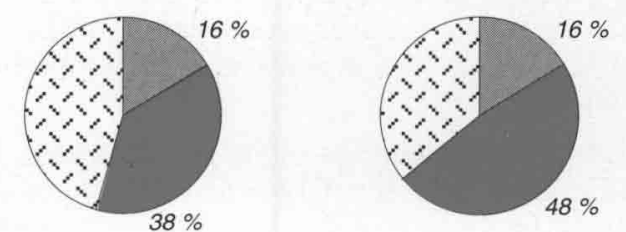

déplacement volontaire

déplacement contraint par l'homme

autres caractéristiques

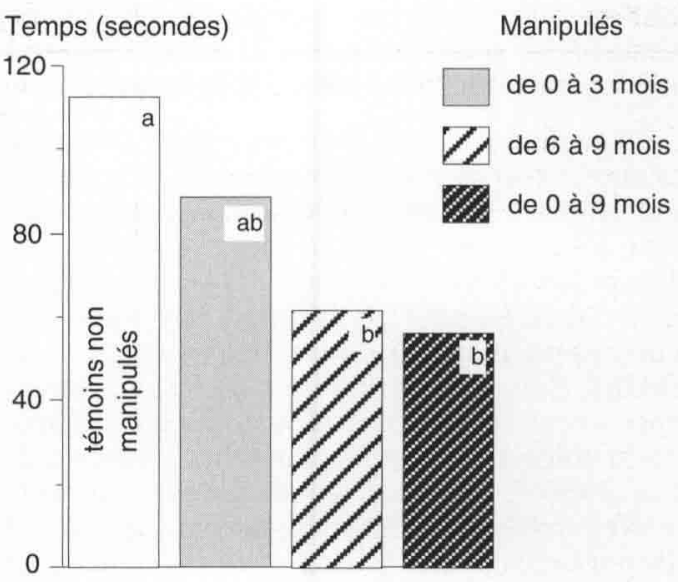

Figure 7 Réactions des animaux en fonction de la période au cours de laquelle ils ont été manipulés (Boissy et Bouissou, 1988). 
Cependant, chez des veaux allaités de race Aubrac, Boivin et al (1992 b)) rapportent un effet bénéfique de manipulations effectuées pendant 10 jours à l'âge d' 1 mois $1 / 2$ alors qu'elles sont sans effet juste après la naissance.

La période du sevrage est, pour certains auteurs, favorable à l'établissement d'une relation Homme-Animal facilitant les manipulations ultérieures : lorsque des porcelets sont manipulés après le sevrage, leurs réactions à l'égard d'un homme ne diffèrent pas d'avec les témoins un mois plus tard (Hemsworth et al 1981a). Par contre, des manipulations effectuées avant sevrage entraînent des effets encore sensibles quatre mois plus tard (Hemsworth et al 1986b). Chez les bovins de race Aubrac, Le Neindre et al (1992) suggèrent également l'importance de la période du sevrage pour faciliter les contacts avec l'Homme.

L'existence, chez les femelles de certaines espèces d'ongulés, d'une période de sensibilité particulière au cours de laquelle se met en place le comportement maternel et s'établit un lien sélectif avec leur jeune aux alentours de la parturition a conduit Hemsworth et al (1987a, 1989) à tenter de mettre à profit cette période pour faciliter la création d'une relation avec l'Homme. Effectivement, la présence d'un humain près de vaches parturientes au cours de l'heure qui suit la mise bas a pour conséquence une plus grande docilité ultérieure de ces animaux lors de la traite. Les vaches ayant eu ce contact avec l'Homme lors de la mise bas présentent, par rapport aux témoins, moins de réactions de peur, approchent plus facilement un humain (à la 6ème semaine de lactation) et présentent des taux de cortisol dans le lait inférieurs.

Enfin, pour certains auteurs, des relations positives entre l'Homme et l'Animal ne pourraient s'établir que par une approche libre de ce dernier, et non par un contact forcé (Hediger 1964). Matéo et al (1991) ont comparé des brebis soumises à 21 jours de contact libre avec un humain ou au contraire à un contact forcé (animal en contention) et des brebis non manipulées. Seules les premières présentent ultérieurement une latence d'approche d'un humain réduite.

\section{Conclusion}

La qualité de la relation Homme-Animal et le comportement de l'éleveur sont des facteurs importants à prendre en compte dans les différents systèmes de production car ils influencent la productivité, la santé et le bien-être des animaux.

Etant donné que les effets négatifs sont le plus souvent consécutifs à des réactions de peur de l'animal et à des manipulations aversives dont un grand nombre pourraient être évitées ou tout au moins minimisées, l'attention de l'éleveur est tout particulièrement requise à ce sujet. Le choix du personnel animalier devrait se faire avec soin et répondre à des critères précis. Cependant, si la modifica- tion du comportement de l'Homme envers les animaux est un aspect des possibilités d'amélioration de la relation Homme-Animal, on peut aussi penser adapter l'Animal à l'Homme et aux diverses techniques utilisées en élevage moderne. Ceci peut se faire, nous l'avons vu, par divers moyens : habituation, apprentissage, manipulations à différentes périodes de la vie de l'animal. A plus longue échéance, il serait possible d'incorporer un index reflétant le "tempérament", la réactivité, ou la peur, dans les programmes de sélection ; ceci permettrait d'obtenir des animaux susceptibles de mieux s'adapter aux contraintes de tel ou tel type d'élevage.

La dernière décennie a vu se développer de nombreuses études concernant l'impact de la relation Homme-Animal sur la productivité. Il est cependant nécessaire d'approfondir nos connaissances dans des domaines tels que la création des attachements interspécifiques, les périodes sensibles, la communication avec l'animal, le "tempérament" et la réactivité, et l'influence de l'expérience ou de la composante génétique. Il restera un effort à faire pour faire passer dans la pratique un certain nombre de concepts et pour changer des attitudes ou habitudes ancestrales.

Enfin, la recherche de techniques alternatives ou susceptibles de minimiser les conséquences néfastes de certains traitements inévitables doit être poursuivie.

On peut ainsi espérer optimiser les productions en respectant le bien-être de l'animal et en facilitant la tâche de l'éleveur.

\section{Références bibliographiques}

ADEYEMO O., HEATH E., 1982. Social behaviour and adrenal cortical activity in heifers. Appl. Anim. Ethol., 8, 99-108.

ARCHER J., 1979. Behavioural aspects of fear. In: "Fear in animals and man", W. Sluckin (Ed.), Van Nostrand Reinhold, New York, pp. 56-85.

BALDOCK N.M., SIBLY R.M., 1990. Effects of handling and transportation on the heart rate and behaviour of sheep. Appl. Anim. Behav. Sci., 28, 15-39.

BARNETT J.L., HEMSWORTH P.H., HAND A.M. 1982/1983. Effects of chronic stress on some blood parameters in the pig. Appl. Anim. Ethol., 9, 273-277.

BARNETT J.L., CRONIN G.M., HEMSWORTH P.H., WINFIELD C.G., 1984. The welfare of confined sows: physiological, behavioural and production responses to contrasting housing systems and handler attitudes. Ann. rech. Vét., 15, 217-226.

BECKER G., EZINGA G., 1969. Early-handling and social rearing effects on dominance-subordination behavior in the adult rat. Psychon. Sci., 15, 2728.

BECKER G., FLAHERTY T.B., 1966. Effects of post weaning tactual stimulation on emotionality and social dominance in the rat. Psychol. Reports, $19,363-366$. 
BECKER G., GAUDET I.J., 1968. Dominancesubordination polarity in early-handled and nonhandled rats. Psychon. Sci., 11, 115-116.

BELYAEV D.K., PLYUSNINA I.Z., TRUT L.N., 1984. Domestication in the silver fox (Vulpes fulvus Desm): changes in physiological boundaries of sensitive period of primary socialization. Appl. Anim. Behav. Sci., 13, 359-370.

BOISSY A., BOUISSOU M.F., 1988. Effects of early handling on heifers'subsequent reactivity to humans and to unfamiliar situations. Appl. Anim. Behav. Sci., 20, 259-273.

BOISSY A., LE NEINDRE P., 1990. Social influences on the reactivity of heifers: implication for learning abilities in operant conditioning. Appl. Anim. Behav. Sci., 25, 149-165.

BOIVIN X., LE NEINDRE P., CHUPIN J.M. GAREL J.P., TRILLAT G., 1992a. Influence of breed and early management on ease of handling and open-field behaviour of cattle. Appl. Anim. Behav. Sci., 32, 313-323.

BOIVIN X., LE NEINDRE P., CHUPIN J.M., 1992b. Establishment of cattle-human relationships. Appl. Anim. Behav. Sci., 32, 325-335.

BOUISSOU M.F., ANDRIEU S., 1978. Etablissement des relations préférentielles chez les bovins domestiques. Behaviour, 64, 148-157.

BOUISSOU M.F., HOVELS J.H., 1976b. Effet d'un contact précoce sur quelques aspects du comportement social des bovins domestiques. Biol. Comp., 1, 17-36.

BRAASTAD B.O., HANSEN I., MELKERAAEN A., BAKKEN M., 1989. Effects of early handling on behaviour and growth in farmed silver foxes. Abst. 21st Intern. Ethol. Conf., Utrecht, 3.

BUCKLAND R.B., GOLDROSEN A., BERNON D.E., 1974. Effect of blood sampling by cardiac puncture on subsequent body weight of broilers and S.C. White Leghorn replacement pullets. Poultry Sci., 53 , 1256-1258

CAIRNS R.B., JOHNSON D.L., 1965. The development of interspecies social attachments. Psychon. Sci., 2, 337-338.

COCKRAM M.S., CORLEY K.T.T., 1991. Effect of pre-slaughter handling on the behaviour and blood composition of beef cattle. Br. vet. J. 147, 444-454.

CSERMELY D., MAINARDI D., SPANO S., 1983. Escape-reaction of captive young red-legged partridges (Alectoris rufa) reared with or without visual contact with man. Appl. Anim. Ethol., 11, 177-182

DALY M., 1973. Early stimulation of rodents: a critical review of present interpretations. Br. J. Psychol., $64,435-460$

DENENBERG V.H., 1963. Early experience and emotional development. Sci. Am., 208, 2-7.

DENENBERG V.H., 1964. Critical periods, stimulus input and emotional reactivity: a theory of infantile stimulation. Psychol. Rev., 71, 335-351

DRYDEN A.L., SEABROOK M.F., 1986. An investigation into some components of the behaviour of the pigstockman and their influence on pig behaviour and performance. J. Agric. Manpower Society, 1, 44-52.
DUNCAN I.J.H., 1990. Reactions of poultry to human beings. In: Zayan R. and Dantzer R. (eds), Social Stress in Domestic Animals, Kluwer Academic Publishers, Dordrecht, pp 121-131.

FELL L.R., SHUTT D.A., 1989. Behavioural and hormonal responses to acute surgical stress in sheep. Appl. Anim. Behav. Sci., 22, 283-294.

FORDYCE G., GODDARD M.E., SEIFERT G.W., 1982. The measurement of temperament in cattle and the effect of experience and genotype. Proc. Aust. Soc. Anim. Prod., 4, 329-332.

FORDYCE G., GODDARD M.E., TYLER R., WILLIAMS G., TOLEMAN M.A., 1985. Temperament and bruising of Bos indicus cross cattle. Aust. J. Exp. Agric., 25, 283-288.

FOX M.W., 1969. Behavioural effects of rearing dogs with cats during the critical period of socialization. Behaviour, 35, 273-280.

FOX M.W., STELZNER D., 1966. Behavioural effects of differential early experience in the dog. Anim. Behav., 14, 273-281.

GALEF B.G.,Jr, 1970. Aggression and timidity: responses to novelty in feral norway rats. J. Comp. Physiol. Psychol., 70, 370-381.

GODDARD M.E., FORDYCE C., HOLROYD R.S., 1983. The measurement and development of "temperament" of cattle. In: "International Symposium of Ethology", Brisbane, Australie, p. 109.

GONYOU H.W., HEMSWORTH P.H., BARNETT J.L., 1986. Effects of frequent interactions with humans on growing pigs. Appl. Anim. Behav. Sci., $16,269-278$

GRANDIN T., 1980a. Bruises and carcass damage. Int. J. Stud. Anim. Probl., 1, 121-137.

GRANDIN T., 1980b. Livestock behavior as related to handling facilities design. Int. J. Stud. Anim. Probl., 1, 33-43

GRANDIN T., 1980c. Observations of cattle behavior applied to the design of cattle-handling facilities. Appl. Anim. Ethol., 6, 19-31.

GRANDIN T., 1984/1985. Race system for cattle slaughter plants with $1.5 \mathrm{~m}$ radius curves. Appl. Anim. Behav. Sci., 13, 295-299.

GRANDIN T., 1987. Design of lairage, yard and race systems for handling cattle in abbatoirs, auctions, ranches, restraining chutes and dipping vats. 1st World Congress on Ethol. Appl. to Zootechnics, Madrid, Spain, pp37-52

GRANDIN T., 1989. Voluntary Acceptance of Restraint by Sheep. Appl. Anim. Behav. Sci., 23, $257-261$.

GRANDIN T., TAYLOR I.A., CURTIS S.E., CARR T.R., 1986. Richness of pig's environment affects handling in chute. Proc. Meeting of Amer. Soc. Anim. Sci., Abstr p161.

GRANDIN T., CURTIS S.E., TAYLOR I.A., 1987. Toys, mingling and driving reduce excitability in pigs. J. Anim. Sci., 65 (suppl 1), 230 (abst).

GROSS W.B., SIEGEL P.B., 1982. Socialization as a factor in resistance to infection, feed efficiency and response to antigen in chickens. Am. J. Vet. Res., 43, 2010-2012 
HARGREAVES A.L., HUTSON G.D., 1990a. The Stress Response in Sheep during Routine Handling Procedures. Appl. Anim. Behav. Sci., 26, 83-90.

HARGREAVES A.L., HUTSON G.D., 1990b Changes in Heart Rate, Plasma Cortisol and Haematocrit of Sheep during a Shearing Procedure. Appl. Anim. Behav. Sci., 26, 91-101.

HARGREAVES A.L., HUTSON G.D., 1990c. An Evaluation of the Contribution of Isolation, UpEnding and Wool Removal to the Stress Response to Shearing. Appl. Anim. Behav. Sci., 26, 103-113.

HARGREAVES A.L., HUTSON G.D., 1990d. The Effect of gentling on Heart Rate, Flight Distance and Aversion of Sheep to a Handling Procedure. Appl. Anim. Behav. Sci., 26, 243-252.

HARGREAVES A.L., HUTSON G.D., 1990e. Some Effects of Repeated Handling on Stress Responses in Sheep. Appl. Anim. Behav. Sci., 26, 253-265.

HEARNSHAW H., BARLOW R., WANT G., 1979 Development of a "temperament" or "handling difficulty" score for cattle. Proc. Aust. Assoc. Anim. Breed. Genet., 1, 164-166.

HEARNSHAW H., MORRIS C.A., 1984. Genetic and environmental effects on a temperament score in beef cattle. Aust. J. Agric. Res., 35, 723-733.

HEDIGER H., 1965. Mensch und Tier im Zoo. Tiergarten Biologie. Albert Muller Verlag, Zurich.

HEIRD J.C, WHITAKER D.D., BELL R.W., RAMSEY C.B., LOKEY C.E., 1986. The effects of handling at different ages on the subsequent learning ability of 2-year-old horses. Appl. Anim. Behav. Sci., $15,15-25$

HEMSWORTH P.H., BARNETT J.L., 1987. Human-Animal interactions. Vet. Clin. North. Americ.: Food Ani. Pract., 3, 339-356

HEMSWORTH P.H., BARNETT J.L., 1991. The effects of aversively handling pigs, either individually or in groups, on their behaviour, growth and corticosteroids. Appl. Anim. Behav. Sci., 30, 61-72.

HEMSWORTH P.H., BARNETT J.L., HANSEN C., 1987a. The influence of inconsistent handling by humans on the behaviour, growth and corticosteroids of young pigs. Appl. Anim. Behav. Sci., 17, 245-252.

HEMSWORTH P.H., BARNETT J.L., COLEMAN G.J., HANSEN C., 1989a. A study of the relationships between the attitudinal and behavioural profiles of stock persons and the level of fear of humans and reproductive performance of commercial pigs. Appl. Anim. Behav. Sci., 23, 301-314.

HEMSWORTH P.H., BARNETT J.L., HANSEN C., 1981a. The influence of handling by humans on the behavior, growth and corticosteroids in the juvenile female pig. Horm. Behav., 15, 396-403

HEMSWORTH P.H., BARNETT J.L., HANSEN C., 1986a. The influence of handling by humans on the behaviour reproduction and corticosteroids of male and female pigs. Appl. Anim. Behav. Sci., 15, 303-314.

HEMSWORTH P.H., BARNETT J.L., HANSEN C., GONYOU H.W., 1986c. The influence of early contact with humans on subsequent behavioural response of pigs to humans. Appl. Anim. Behav. Sci., $15,55-63$
HEMSWORTH P.H., BARNETT J.L., TILBROOK A.J., HANSEN C., 1989b. The effects of handling by humans at calving and during milking on the behaviour and milk cortisol concentrations of primiparous dairy cows. Appl. Anim. Behav. Sci., 22, 313-326.

HEMSWORTH P.H., BARNETT J.L., TREACY D. MADGWICK P., 1990. The Heritability of the Trait Fear of Humans and the Association Between This Trait and Subsequent Reproductive Performance of Gilts. Appl. Anim. Behav. Sci., 25, 85-95.

HEMSWORTH P.H., BRAND A., WILLEMS P. $1981 \mathrm{~b}$. The behavioural response of sows to the presence of human beings and its relation to productivity. Livest. Prod. Sci., 8, 67-74.

HEMSWORTH P.H., GONYOU H.W., DZIUK P.J., 1986 b. Human communication with pigs: the behavioural response of pigs to specific human signals. Appl. Anim. Behav. Sci., 15, 45-54.

HEMSWORTH P.H., HANSEN C., BARNETT J.L., 1987b. The effects of human presence at the time of calving of primiparous cows on their subsequent behavioural response to milking. Appl. Anim. Behav. Sci., 18, 247-255.

HEMSWORTH P.H., BARNETT J.L., COLEMAN G.J., 1992. Fear of humans and its consequences for the domestic pig. In: The inevitable bond Examining scientist-animal interactions. Davis H., Balfour D. eds. Cambridge University Press. 264-284.

HUGHES B.O., BLACK A.J., 1976. The influence of handling on egg production, egg shell quality and avoidance behaviour of hens. Br. Poult. Sci., 17, 135144

HUTSON G.D., 1982. "Flight distance" in merino sheep. Anim. Prod., 35, 231-235.

HUTSON G.D., 1985. The influence of barley food rewards on sheep movement throught a handling system. Appl. Anim. Behav. Sci., 14, 263-273.

JEPHCOTT E.H., McMILLEN I.C., RUSHEN J., HARGREAVES A., THORBURN G.D., 1986. Effect of electroimmobilisation on ovine plasma concentrations of $\beta$-endorphin/ $\beta$-lipotrophin, cortisol and prolactin. Res. Vet. Sci., 41, 371-377.

JONES R.B., 1983. Fear responses in chicks as a function of the social environment. Behav. Process., $8,309-325$.

JONES R.B., 1984. Open-field responses of domestic chicks in the presence of a congenate or a strange chick. I. R. C. S. Med. Sci., 12, 482-483.

JONES R.B., 1985. Fear responses of individuallycaged laying hens as a function of cage level and aisle. Appl. Anim. Behav. Sci., 14, 63-74.

JONES R.B., 1987. Fearfulness of cage laying hens: the effects of cage level and type of roofing. Appl. Anim. Behav. Sci., 17, 171-175.

JONES R.B., 1987. The assessment of fear in the domestic fowl. In: "Cognitive aspects of social behaviour in the domestic fowl", R. ZAYAN, I.J.H. DUNCAN (Eds.), Elsevier, Amsterdam, pp. 40-81.

JONES R.B., MERRY B.J., 1988. Individual or paired exposure of domestic chicks to an open-field: some behavioural and adrenocortical consequences. Behav. Process., 16, 75-86.

JONES R.B., FAURE J.M., 1981. The effects of regular handling on fear in the domestic chick. Behav. Process., 6, 135-143. 
JONES R.B., HUGHES B.O., 1981. Effects of regular handling on growth in the male and female of broiler and layer strains. Br. Poult. Sci., 22, 461-465.

KALLWEIT E., UNSHELM J., WEMKEN K., SMIDT D., 1980. Effects of transportation and preslaughter holding on some blood parameters and on meat quality in fattening bulls. In: "EEC Seminar Problem of Dark-Cutting in Beef", Bruxelles, Belgique, p. 16.

KERR S.C.G., WOOD-GUSH D.G.M., 1987. The development of behaviour patterns and temperament in dairy heifers. Behav. Process., 15, 1-16.

KERSTEN A.M.P., MEIJSSER F.M., METZ J.H.M., 1989. Effects of early handling on later openfield behaviour in rabbits. Appl. Anim. Behav. Sci., $24,157-167$

KILGOUR R., DE LANGEN H., 1970. Stress in sheep resulting from management practices. Proc. N.Z. Soc. Anim. Prod., 30, 65-76.

LYONS D.M. 1989. Individual differences in temperament of dairy goats and the inhibition of milk ejection. Appl. Anim. Behav. Sci., 22, 269-282.

LYONS D.M. 1992. Early human-animal relationships and temperament differences among domestic dairy goats. In: The inevitable bond Examining scientist-animal interactions. Davis H., Balfour D. eds. Cambridge University Press. 295-315.

LYONS D.M., PRICE E.O., 1987. Relationships between heart rates and behavior of goats in encounters with people. Appl. Anim. Behav. Sci., 18, 363-369.

LYONS D.M., PRICE E.O., MOBERG G.P., 1988a. Individual differences in temperament of domestic dairy goats: constancy and change. Anim. Behav., $36,1323-1333$

LYONS D.M., PRICE E.O., MOBERG G.P., 1988b. Social modulation of pituitary-adrenal responsiveness and individual differences in behavior of young domestic goats. Physiol. Behav., 43, 451-458.

MATEO J.M., ESTEP D.Q., McCANN J.S., 1991. Effects of differential handling on the behaviour of domestic ewes (Ovis aries). Appl. Anim. Behav. Sci., $32,45-54$.

McCANN J.S., HEIRD J.C., BELL R.W., LUTHERER L.O., 1988a. Normal and more highly reactive horses. I. Heart rate, respiration rate and behavioral observations. Appl. Anim. Behav. Sci., 19, 201-214.

McCANN J.S., HEIRD J.C., BELL R.W. LUTHERER L.O., 1988b. Normal and more highly reactive horses. II. The effect of handling and reserpine on the cardiac response to stimuli. Appl. Anim. Behav. Sci., 19, 215-226.

MEANEY M.J., AITKEN D.H., BODNOFF S.R., INY L.J., TATAREWICZ J.E., SAPOLSKY R.M., 1985. Early postnatal handling alters glucocorticoid receptor concentrations in selected brain regions. Behav. Neurosci., 99, 765-770.

METZ J.H.M., 1983. Effects of early handling in the domestic rabbit. Appl. Anim. Ethol., 11, 86-87 (Abstract).

METZ J.H.M., 1987. The response of farm animals to humans. Examples of the epistemology of experimental research. In : The role of the stockman in livestock productivity and management. Proc. EEC Seminar, Bruxelles 16-17 Dec 1986, 23-37.

MEZEI T.C., ROSEN J., 1960. Dominance behavior as a function of infantile stimulation in the rat. Arch. Gen. Psychiat., 3, 53-56.
MOBERG G.P., 1987. A model for assessing the impact of behavioral stress on domestic animals. J. Anim. Sci., 65, 1228-1235.

MOBERG G.P., ANDERSON C.O., UNDERWOOD T.R., 1980. Ontogeny of the adrenal and behavioral responses of lambs to emotional stress. J. Anim. Sci., $51,138-142$

MOBERG G.P., WOOD V.A., 1982. Effect of differential rearing on the behavioral and adrenocortical response of lambs to a novel environment. Appl. Anim. Ethol., 8, 269-279.

MORMEDE P. DANTZER R., BLUTHE R.M., CARITEZ J.C., 1984. Differences in adaptative abjlities of three breeds of Chinese pigs. Behavioural and neuroendocrine studies. Génét. Sél. Evol., 16, $85-102$.

MURPHEY R.M., MOURA DUARTE F.A., TORRES PENEDO M.C., 1980. Approachability of bovine cattle in pastures: breed comparisons and a breed $\mathrm{x}$ treatment analysis. Behav. Genet., 10, 171-181.

MURPHEY R.M., MOURA DUARTE F.A., TORRES PENEDO M.C., 1981. Responses of cattle to humans in open spaces: breed comparisons and approach avoidance relationships. Behav. Genet., $11,37-48$.

MURPHY L.B., 1978. The practical problem of recognizing and measuring fear and exploration behaviour in the domestic fowl. Anim. Behav., 26, 461465 .

MURPHY L.B., DUNCAN I.J.H., 1977. Attempts to modify the responses of domestic fowl towards human beings. I. The association of human contact with a food reward. Appl. Anim. Ethol., 3, 321-334.

MURPHY L.B., DUNCAN I.J.H., 1978. Attempts to modify the responses of domestic fowl towards human beings. II. The effect of early experience. Appl. Anim. Ethol., 4, 5-12.

NEREM R.M., LEVESQUE M.J., CORNHILL J.F., 1980. Social environment as a factor in diet-induced atherosclerosis. Science, 208, 1475-1476.

PATERSON A.M., PEARCE G.P., 1989. Boar-induced puberty in gilts handled pleasantly or unpleasantly during rearing. Appl. Anim. Behav. Sci., 22, 225-233.

PEARCE G.P., PATERSON A.M., PEARCE A.N., 1989. The Influence of Pleasant and Unpleasant Handling and the Provision of Toys on the Growth and Behaviour of Male Pigs. Appl. Anim. Behav. Sci., 23, 27-37.

PEDERSEN V., JEPPESEN L.L., 1990. Effects of Early Handling on Later Behaviour and Stress Responses in the Silver Fox (Vulpes vulpes). Appl. Anim. Behav. Sci., 26, 383-393.

PODBERSCEK A.L., BLACKSHAW J.K., BEATTIE A.W., 1991. The effects of repeated handling by familiar and unfamiliar people on rabbits in individual cages and group pens. Appl. Anim. Behav. Sci., $28,365-373$.

PRICE E.O., WALLACH S.J.R., 1990. Physical isolation of hand-reared Hereford bulls increases their aggressiveness toward humans. Appl. Anim. Behav. Sci., 27, 263-267.

REID R.L., MILLS S.C., 1962. Studies on the carbohydrate metabolism of sheep. XIV: The adrenal response to psychological stress. Aust. J. Agric. Res., $13,282-295$. 
ROMEYER A., BOUISSOU M.F., 1992. Assessment of fear reactions in domestic sheep, and influence of breed and rearing conditions. Appl. Anim. Behav. Sci. 34, 93-119.

ROSEN J., 1958. Dominance behavior as a function of postweaning gentling in the albino rat. Canad. J. Psychol. Bull., 57, 476-492

RUBIO I., BLACKSHAW J.K., CHENOWETH P.J., BEATTIE A.W., 1988. A preliminary study on dominance, temperament and cortisol levels in Droughtmaster heifers. Proc. Aust. Soc. Anim. Prod., 17,465 .

SAMBRAUS H.H., UNSHELM J., 1983. Influence of man on behaviour and production of animals. Proc. E.A.A.P. meeting, Madrid., 13pp.

SATO S., SEKI Y., KUROSAKI Z., 1983. Factor analysis of tractability of dairy cattle. Proc. Vth Wld Conf. Anim. Prod. Tokyo, 2, 817-818.

SATO S., SHIKI H., YAMASAKI F., 1984. The effects of early caressing on later tractability of calves. Jpn. J. Zootech. Sci., 55, 332-338.

SCHAEFER Jr.T., 1963. Early "experience" and its effects on later behavioral processes in rats: II-a critical factor in the early handling phenomenon. Trans. N.Y. Acad. Sci., 25, 871-889.

SCHLICHTING M.C., SMIDT D., 1987. The influence of the stockman on the milk yield of cows. In : The role of the stockman in livestock productivity and management. Proc. EEC Seminar, Bruxelles 16-17 Dec 1986, 53-58.

SCOTT J.P., 1962. Critical periods in behavioral development. Science, 138, 949-958.

SCOTT J.P., 1978. Critical periods for the development of social behaviour in dogs. In: "Critical Period", J.P. SCOTT (Ed.), Dowden, Hutchinson and Ross, Stroudsburg, PA, pp. 186-196.

SEABROOK M.F., 1972. A study to determine the influence of the herdman's personality on milk yield. J. Agric. Labour Sci., 1, 1-45.

SEABROOK M.F., 1984a. The personality of the dairy stockman and its influence on the interaction process. Proc. Int. Congress on Applied Ethology in farm animals. KIEL. Unshelm J, van Putten G. Zeeb K. Eds. 245-250.
SEABROOK M.F., 1984b. The psychological interaction between the stockman and his animals and its influence on performance of pigs and dairy cows. Vet. Rec., 115, 84-87.

SEABROOK M.F., 1987. The role of the stockman in livestock productivity and management. Research epistemology: The holistic approach. In : The role of the stockman in livestock productivity and management. Proc. EEC Seminar, Bruxelles 16-17 Dec 1986, 39-51.

SEABROOK M.F., 1990. Reactions of dairy cattle and pigs to human beeings. In: Zayan R. and Dantzer R., (eds), Social Stress in Domestic Animals, Kluwer Academic Publishers, Dordrecht, pp 110-120.

SEABROOK M.F., BARTLE N.C., 1991. Environmental factors influencing the production and welfare of farm animals. Int. Conf. on Farm Animals and the Environment, 5-6 September Bangor, Wales, U.K.

TENNESSEN T., 1988. Effect of early handling on open-field behaviour and fear of humans in young silver fox (Vulpes vulpes). In: "International Congress on Applied Ethology in Farm Animals", Skara, Suède, p. 87 (abstr.).

THOMPSON C.I., 1976. Growth in the Hubbard broiler: increased size following early handling. Dev. Psychobiol., 9, 459-464.

TORRES-HERNANDEZ G., HOHENBOKEN W., 1979. An attempt to assess traits of emotionality in crossbred ewes. Appl. Anim. Ethol., 5, 71-83.

UNSHELM J., 1987. The role of the stockman in livestock productivity and management. Introductory comments. In : The role of the stockman in livestock productivity and management. Proc. EEC Seminar, Bruxelles 16-17 Dec 1986, 3-9.

VEISSIER I., 1987. Capacités comportementales d'adaptation des bovins à de nouvelles conditions de vie. Thèse Doc. Univ. Rennes 1, 146pp.

WARRISS P.D., 1990. The handling of cattle preslaughter and its effects on carcass and meat quality. Appl. Anim. Behav. Sci., 28, 171-186.

WHITTLESTONE W.G., KILGOUR R., de LANGEN H., DUIRS G., 1969. Behavioral stress and the cell count of bovine milk. J. Milk Food Technol., $33,217-220$.

\section{Summary}

\section{Human-Animal relationships : consequences and possibilities for improvement.}

The quality of Human-Animal relationships and the attitude of the stock-person are important factors to take into account in the various production systems as they have an impact on productivity, health and welfare of the animals.

A poor Human-Animal relationship is mainly reflected in fear reactions of the animals. Various methods to evaluate fear in the presence of a human have been developped; they include behavioural (flight distance, latency to approach, time spent near a human, reactions to handling, etc...) as well as physiological (heart rate, cortisol levels) criteria. Human-Animal relationship is influenced by rearing conditions, environmental factors, experience, nature of previous contacts, genetic factors and finally by attitude and personality of the stock-person. A poor Human-Animal relationship results in stress reactions, which is turn lead to a reduction in production.

It is possible to improve Human-Animal relation. ships through modification of the stock-person's attitude, by means of habituation and learning processes, and by the establishment of bonds during sensitive periods of the animal's life.

BOUISSOU M.-F., 1992. La relation Homme-Animal. Conséquences et possibilités d'amélioration. INRA Prod. Anim. 5 (5), 303-318. 